\title{
AN IDENTITY WITH APPLICATIONS TO HARMONIC MEASURE
}

BY DAVID S. JERISON 1 AND CARLOS E. KENIG 2

In this note we use an elementary integral formula to give a new short proof of the theorem of B. E. J. Dahlberg [2] on the mutual absolute continuity of harmonic and surface measure on bounded Lipschitz domains. (See [4] for the relevant definitions.) The formula also provides a new proof of the so-called reverse Holder inequality (see [2]), and $L^{p}$-estimates for the Dirichlet problem (see [3]). Furthermore, we are able to treat domains whose boundaries are worse than Lipschitz. In particular, we show that if $D \subset R^{n}$ is given locally (in the appropriate sense) by the graph of a function $\phi$ with $\nabla \phi \in L^{p}, p>n-1$, and $D$ is regular for the Dirichlet problem, then harmonic measure and surface measure on $\partial D$ are mutually absolutely continuous. The hypothesis of regularity is superfluous when $n=3$, or under the stronger assumption $\nabla \phi \in \mathrm{BMO}$, but not necessarily otherwise.

We wish to thank Professor E. M. Stein for many helpful conversations.

Theorem 1. Let $D$ be a bounded Lipschitz domain in $\mathbf{R}^{n}$. Let $\omega$ be harmonic measure of $\partial D$ with respect to $X_{0} \in D$. Then

(a) $\omega$ and $\sigma$ are mutually absolutely continuous.

(b) Let $k(Q)=d \omega / d \sigma$. Then $k \in L^{2}(d \sigma)$; moreover, for every surface ball $\Delta \subset \partial D$,

$$
\left(\frac{1}{\sigma(\Delta)} \int_{\Delta} k^{2}(Q) d \sigma(Q)\right)^{1 / 2} \leqslant \frac{c}{\sigma(\Delta)} \int_{\Delta} k(Q) d \sigma(Q) .
$$

To establish our theorem, we need

Lemma 1 (The main identity). Assume that $D$ is a bounded $C^{\infty}$ domain in $\mathbf{R}^{n}$, containing 0 . Let $\omega$ be harmonic measure of $\partial D$ at 0 . Then if $k=$ $d \omega / d \sigma$,

$$
\frac{1}{\omega_{n}} \int_{\partial D} \frac{k(Q)}{|Q|^{n-2}} d \sigma(Q)=\int_{\partial D} k^{2}(Q)\left\langle Q, N_{Q}\right\rangle d \sigma(Q),
$$

where $N_{Q}$ is to outer unit normal, and $\omega_{n}$ the surface area of $S^{(n-1)}$.

Received by the editors November 16, 1979.

AMS (MOS) subject classifications (1970). Primary 31 BXX, 31 B05, $31 \mathrm{~B} 25$.

1 The first author was partly supported by an NSF graduate fellowship.

2 The second author was supported in part by an NSF grant. 
Proof. Let $G(X)$ be the Green function of $D$ with pole at 0 . Then

$$
G(X)=1 /|X|^{n-2}-u(x),
$$

where $u$ is harmonic in $D$ and $G$ is 0 on $\partial D$. Moreover, $k(Q)=$ $-\left(1 /(n-2) \omega_{n}\right)\left(\partial G / \partial N_{Q}\right)(Q)$. Let $\alpha=Q-N_{Q}\left\langle Q, N_{Q}\right\rangle$. Because $\left\langle\alpha, N_{Q}\right\rangle=0$, $\sum_{i=1}^{n} \alpha_{i}(Q)\left(\partial G / \partial x_{i}\right)(Q)=0$. Thus $\int_{\partial D} k(Q)\left(\sum_{i=1}^{n} \alpha_{i}(Q)\left(\partial G / \partial x_{i}\right) d \sigma(Q)\right)=0$. Hence

$$
-\int_{\partial D} k(Q)\langle Q, \nabla G(Q)\rangle d \sigma(Q)=(n-2) \omega_{n} \int_{\partial D} k^{2}(Q)\left\langle Q, N_{Q}\right\rangle d \sigma(Q) .
$$

However, if $v(x)=x \nabla u(x)$, then $v$ is harmonic in $D$ and $v(0)=0$. Thus $\int_{\partial D} k(Q)\langle Q, \nabla u(Q)\rangle d \sigma(Q)=0$ and our identity follows.

By [4, p. 320], and the definition of Lipschitz domain, we note that it is enough to prove Theorem 1(a) for starshaped Lipschitz domains $\Omega$, with the additional property that they can be exhausted by star-shaped $C^{\infty}$ domains with comparable Lipschitz constants, and with boundaries in a 1-1 correspondence radially. Since $\Omega, \Omega_{j}$ are regular for the Dirichlet problem, it is easy to see that if $\omega, \omega_{j}$ are the respective harmonic measures, $\omega_{j} \rightarrow \omega$ weakly. On the other hand, if we apply Lemma 1 to $\Omega_{j}$, we see that $(\dagger) \int_{\partial \Omega_{j}} k_{j}^{2}(Q) \leqslant c$ where $c$ depends only on the Lipschitz constant of $\Omega$. It then follows that there exists an $L^{2}(d \sigma)$ function $k$, satisfying $(\dagger)$ such that $d \omega=k d \sigma$. To prove that $\sigma \ll \omega$, we notice that once $(\dagger)$ is established, the elementary argument in [2, p. 280], can be repeated.

For the proof of Theorem 1(b), choose coordinates $(x, y), x \in \mathbf{R}^{n-1}$, $y \in \mathbf{R}$, so that near a point $Q_{0}$ of $\partial D, D$ is the region above the graph of a Lipschitz function $\phi$ and $\Delta=\Delta\left(Q_{0}, r\right)=\left\{(x, \phi(x)) \in \partial D /\left|x-x_{0}\right|<r\right\}$. Let $A$ be a point above $\Delta$, in $D$, with $\operatorname{dist}(A, \Delta) \simeq C r$. (*) follows from the estimates

(1) $\int_{\Delta} k_{A}^{2} d \sigma(Q) \leqslant c / \sigma(\Delta)$,

(2) $k(Q) \leqslant c k_{A}(Q) \omega(\Delta)$ a.e. $Q \in \Delta$,

where $k_{A}(Q)=d \omega^{A} / d \sigma$. (2) follows from the estimate at the bottom of p. 512 in [5]. Estimate (1) has an easy proof when $D$ is a special kind of domain. Suppose that $D$ can be approximated in a regular way by $C^{\infty}$ domains $\Omega_{j}$ and that

$$
\begin{gathered}
\operatorname{dist}\left(A, \partial \Omega_{j}\right) \simeq r . \\
\left\langle A-Q_{j}, N_{Q_{j}}\right\rangle \geqslant c r \text { a.e. } Q_{j} \in \Delta_{j}\left(\Delta_{j} \text { corresponds to } \Delta\right) . \\
\left\langle A-Q_{j}, N_{Q_{j}}\right\rangle \geqslant 0 \text {, a.e. } Q_{j} \in \partial \Omega_{j}\left(\Omega_{j} \text { is star-shaped w.r.t. } A\right) .
\end{gathered}
$$

The main identity, (3), (4) and (5) yield

$$
\int_{\Delta_{j}} k_{A}^{j}(Q)^{2} d \sigma_{j}(Q) \leqslant c r^{1-n},
$$

and a passage to the limit gives (1). 
(b) Moreover, if $p>n-1$, then $\sigma \ll \omega$.

The proof of Theorem 3(a) is similar to that of Theorem 1(a). The only difference is that we apply Holder's inequality before invoking the main identity. For part (b) we use (see [6, Chapter 8]).

LEMmA. If $\phi \in L_{1}^{p}\left(R^{n-1}\right), p>n-1$, and $\phi$ is nonnegative and supported in a cube $Q$, then for any $\epsilon>0$ there is a closed set $F$ and a Lipschitz function $\psi$ supported in $Q$ such that $|Q-F|<\epsilon, \phi=\psi$ on $F$ and $\phi \geqslant \psi \geqslant 0$.

We will call a domain $D$ in $\mathbf{R}^{n}$ locally interior (resp. exterior) star-shaped if there exist closed sets $F_{i}$ and open sets $V_{i}$ with $F_{i} \subset V_{i}, \partial D=\bigcup F_{i}$ and points $X_{i} \in \mathbf{R}^{n}$ and continuous functions $\phi_{i}$ on $S^{(n-1)}, 0<c \leqslant \phi_{i}$, such that in polar coordinates with $X_{i}$ as origin,

$$
D \cap V_{i}=\left\{(r, \theta) \mid \theta \in S^{n-1}, 0 \leqslant r<\phi_{i}(\theta)\right\} \cap V_{i}
$$

and

$$
F_{i} \subset\left\{\left(\phi_{i}(\theta), \theta\right) \mid \theta \in S^{n-1}\right\}
$$

(resp. $\left\{(r, \theta) \mid 0 \leqslant r<\phi_{i}, \theta \in O_{i}\right.$, a spherical cap in $\left.S^{(n-1)}\right\} \subset{ }^{c} \bar{D}$ and $F_{i} \subset$ $\left.\left\{\left(\phi_{i}(\theta), \theta\right) \mid \theta \in O_{i}\right\}\right)$.

Corollary. Let $D$ be a bounded domain, regular for the Dirichlet problem:

(a) If $D$ is locally interior star-shaped and $\nabla \phi_{i} \in L^{p}(d \theta), p>2$, then $\omega \ll$ o. If $p>n-1$, then $\sigma \ll \omega$.

(b) If $D$ is locally exterior star-shaped, then in addition to (a) we have, $k \in L^{q}(d \sigma)$ for $q=2(p-1) / p$.

The corollary follows easily from Theorem 3. To deduce part (b), we apply the Kelvin transform.

Some consequences of Wiener's criterion [7] are:

(1) When $n=3$, the sole hypothesis $\left|\nabla \phi_{j}\right| \in L^{p}(d \theta)$ implies $D$ is regular;

(2) When $n \geqslant 4$, a region with $|\nabla \phi| \in L^{p}(d \theta)$ for all $p<\infty$ may fail to be regular. However, when $\nabla \phi_{j} \in \mathrm{BMO}, D$ is regular since $\phi_{j} \in \Lambda_{*}$, the Zygmund class. Another sufficient condition for regularity is

$$
\phi_{j} \in \operatorname{Lip}\left(h(\log 1 / h)^{1 /(n-3)}\right) .
$$

The exponent $1 /(n-3)$ is sharp. An example for which regularity fails is the domain below the graph of $\phi(x)=|x|(\log 1 /|x|)^{\alpha}, x \in \mathbf{R}^{n-1}, \alpha>1 /(n-3)$. Note that $\nabla \phi \in L_{\text {loc }}^{p}(d x)$ for all $p<\infty$ and $\phi \in \operatorname{Lip}\left(h(\log 1 / h)^{\alpha}\right)$. 
We can reduce the general case to this special case by replacing $D$ with a larger, unbounded region $D^{\prime}$ with $\Delta \subset \partial D \cap \partial D^{\prime}$. We then take the Kelvin transform $K(X)=\widetilde{X}=(X-R) /|X-R|^{2}$ with respect to a point $R \in E={ }^{c} D^{\prime}$. Let $d \widetilde{\sigma}$ be surface measure on $K\left(\partial D^{\prime}\right)$ and let $\widetilde{k}_{A}(\widetilde{Q}) d \widetilde{\sigma}(\widetilde{Q})$ be the harmonic measure at $\widetilde{A}$ for $K\left(D^{\prime}\right) \cup\{0\}$. If, $\operatorname{dist}\left(R, D^{\prime}\right) \geqslant c_{0}$, then $d \widetilde{\sigma}$ and $d \sigma$ are comparable. If $\widetilde{u}$ is harmonic in $K\left(D^{\prime}\right)$, then $|X-R|^{(2-n)} \widetilde{u}(\widetilde{X})$ is harmonic in $D^{\prime}$. Therefore the maximum principle shows that $k_{A}(Q) \leqslant c \widetilde{k}_{A}^{\sim}(\widetilde{Q})$. Thus it suffices to prove the analogue of (1) for $\widetilde{k}, \widetilde{\Delta}$ and $\widetilde{\sigma}$. We now define $R, A, D^{\prime}$ precisely. For sufficiently small $r$, there exists $R=\left(x_{0}, y_{1}\right) \in{ }^{c} D$ such that $\operatorname{dist}(R, \Delta) \geqslant$ $c_{1}$, and such that $\widetilde{\Delta}$ is the graph of a Lipschitz function with norm dominated by that of $\phi$. This follows from the inverse function theorem, and the fact that the Jacobian $\left(a_{i j}\right)$ of $K$ near $\Delta$ can be written as

$$
a_{i j}= \begin{cases}c_{1}^{-2} \delta_{i j}+O(r), & i, j \leqslant n-1 \\ -c_{1}^{-2} \delta_{i j}+O(r), & i=n \text { or } j=n, \text { where } x_{n}=y .\end{cases}
$$

Hence, we can choose $A=\left(x_{0}, y_{0}\right) \in D$ so that the analogue of (3) and (4) hold. Define $E=\left\{X \mid X=t(Q-A)+A\right.$, for $\left.Q \in \Delta, t \geqslant 1 ;|X-A| \leqslant c_{5}\right\}$, $D^{\prime}={ }^{c} E$. We can assume $D \subset D^{\prime}$ and $\operatorname{dist}\left(R, \partial D^{\prime}\right) \underset{\sim}{\sim} c_{0} . K\left(D^{\prime}\right) \cup\{0\}$ is starshaped with respect to $\widetilde{A}$ because the line through $\widetilde{A}, \widetilde{Q}$ corresponds under the (inverse) Kelvin transformation to the circle (or line) through $A, Q$, and $R$. Thus (5) is valid and we are reduced to the special case.

THEOREM 2. If $f \in L^{p}(d \sigma), p \geqslant 2$, then $f$ has a harmonic extension $u$ in $D$, with nontangential maximal function $N(u) \in L^{p}(d o)$. Also, u converges nontangentially to $f$ a.e. $d \sigma$.

Proof. The reduction in the proof of Theorem 1(b) shows that we may as well assume $D$ is star-shaped. Let

$$
M(f)(Q)=\sup _{Q \in \Delta} \frac{1}{\omega(\Delta)} \int_{\Delta} f(\widetilde{Q}) d \omega(\widetilde{Q}) .
$$

By [4, p. 315], $N(u)(Q) \leqslant c M f(Q)$ a.e. $d \sigma$. The result now follows from Theorem 1(b) and the weighted norm inequalities of Muckenhoupt et al (see [1]).

FURTHER RESUlTS. Let $\Omega$ be a star-shaped domain given by

$\Omega=\left\{(r, \theta) \mid 0 \leqslant r<\phi(\theta), \phi(\theta) \geqslant c>0 ; \phi\right.$ continuous, $\left.\theta \in S^{(n-1)}\right\}$.

THEOREM 3. Suppose that $\Omega$ is regular for the Dirichlet problem, and starshaped as above.

(a) If $\nabla \phi \in L^{p}(d \theta), p>2$, then $\omega \ll \sigma$ and

$$
k=d \omega / d \sigma \in L^{q}(d \sigma) \quad \text { for } q=2(p-1) / p .
$$




\section{BIBLIOGRAPHY}

1. R. R. Coifman and C. Fefferman, Weighted norm inequalities for maximal functions and singular integrals, Studia Math. 51 (1974), 241-250.

2. B. E. J. Dahlberg, Estimates of harmonic measure, Arch. Rational Mech. Anal. 65 (1977), 272-288.

3. On the Poisson integral for Lipschitz and $C^{1}$ domains, Studia Math. (to appear).

4. R. Hunt and R. L. Wheeden, , On the boundary values of harmonic functions, Trans. Amer. Math. Soc. 132 (1968), 307-322.

5. - Positive harmonic functions on Lipschitz domains, Trans. Amer. Math. Soc. 147 (1970), 507-527.

6. E. M. Stein, Singular integrals and differentiability properties of functions, Princeton Univ. Press, Princeton, N.J., 1970.

7. N. Wiener, The Dirichlet problem, J. Math. Phys. 3 (1924), 127-146.

DEPARTMENT OF MATHEMATICS, PRINCETON UNIVERSITY, PRINCETON, NEW JERSEY 08544 